\title{
Nicolas Pien*
}

\section{Retrouver la lumière: Métaphysique dans Le Déluge de J.M.G. Le Clézio}

DOI: http://dx.doi.org/10.12775/LC.2020.024

Résumé: Les premiers romans de Le Clézio sont traversés par la question métaphysique, dans une époque devenue athée. Fortement influencé par Saint-Augustin, et par Plotin, Le Déluge - dont le titre est le reflet de l'ambition "biblique" de l'auteur -, publié en 1966, fait état de la recherche d'une "vérité", d'une lumière dans une réalité devenue "spectacle" et factice, recherche qui pose, in fine, la question de la grâce dans la société des "trente glorieuses" auscultée et dénoncée. Après avoir recherché, dans la ville, le mal contemporain - un monde d'artifices où le langage et la lumière se perdent - Besson, le "héros", si proche de Le Clézio, propose une façon d’aller vers un bien qu'il définit dans la dernière partie du roman. Il s'agira donc de confronter Le Déluge aux principes augustiniens afin de comprendre en quoi le texte envisage un itinéraire vers la grâce ou vers une forme de vérité et en quoi Le Clézio y définit aussi une mission de l'écriture et de l'écrivain au $\mathrm{XX}^{\mathrm{e}}$ siècle qui s'incarnera plus tard dans Désert (1980), par exemple.

Mots clés: Déluge, Saint-Augustin, Plotin, Vérité, Grâce

* Docteur en Littérature française de I'Université de Caen-Normandie; enseignant, a écrit sur Le Clézio, Duras, Beckett, Toussaint et, également, sur les littératures antillaises.

E-mail: nicolas.pien@unicaen.fr | ORCID: 0000-0002-9638-9342. 


\title{
Finding the Light \\ Metaphysics in Le Déluge de J.M.G. Le Clézio
}

\begin{abstract}
In an era that has become atheistic, the metaphysical question is a fundamental aspect within the first novels by Le Clézio. Published in 1966 and strongly influenced by Saint Augustine, and by Plotinus, Le Déluge - its title being a reflection of the author's "biblical" ambition - , describes the search for a "truth", for a light in a reality that has become an artificial "show", a research that ultimately deals with the question of grace in the examined and denunciated society of the "Trente Glorieuses". After having searched the city for contemporary evil, - the city, a world of artifices where language and light get lost - Besson, the "hero", quite close to Le Clézio, offers a way to turn towards a good which he defines in the last part of the novel. It will therefore be a question of confronting Le Déluge with Augustinian principles in order to understand how the text envisages a route towards grace or towards a form of truth. Le Clézio also defines a mission to writing and to the writer of the XXth century which will be embodied later, for example in Désert (1980).
\end{abstract}

Keywords: Deluge, Saint Augustine, Plotinus, Truth, Grace

Justyna Gambert qui l'interroge sur ses lectures au début des années 60, Le Clézio répond qu'elles sont essentiellement celles de sa classe de philosophie, Parménide, Platon et Plotin, et celles, incontournables, de son temps, Camus et Sartre, entre autres, qui entérinent l'idée d'un ciel devenu vide. L'Étranger, dans la scène célèbre de l'assassinat de l'Arabe, puis Le Mythe de Sisyphe ont dit et redit un ciel vidé de sa substance métaphysique, d'un ciel qui ne serait que lumière et uniquement lumière. Comme chez Beckett. Comme chez Cioran. Comme chez bien d'autres écrivains. À bien des égards, la question du "ciel”, celle de son sens, est essentielle dans cette littérature qui s'écrit au lendemain de la Seconde Guerre mondiale: c'est dans ce contexte que Le Clézio débute en littérature, bien plus concerné par cette question qu'on ne l'imagine.

À ce "ciel vidé", Camus répond par la "révolte métaphysique", tandis que Sartre répond par "l'existentialisme", deux concepts mus par un même principe, celui de la "conscience" dont on sait qu'il fut au cœur de l'interrogation du jeune Le Clézio. Pourtant, et même si l'influence de La Nausée sur Le Procès-verbal est notable, Le Clézio ne semble pas adopter l'une ou l'autre de ces réponses. Pas de révolte solidaire chez lui, bien au contraire, Adam Pollo finit seul dans sa chambre d'asile, pas de "choix sartrien" non plus: Adam Pollo ne choisit en rien ce qu'il peut devenir, bien au contraire encore, il subit. Notons, cependant, que Le Déluge, annoncé à l'éditeur dès la fin de la lettre qui a accompagné le manuscrit chez Gallimard ("Enfin, je me permets de vous signaler que j’ai entrepris la rédaction d'un autre récit, beaucoup plus étendu, racontant avec le maximum de simplicité ce qui se passe le lendemain de la mort d'une jeune fille" (Le Clézio 1963: 13), reprend un questionnement camusien: Anna, la jeune fille qui parle sur la bande magnétique que Besson écoute au chapitre I. se suicide pour une banale histoire d'amour. Inutile de repréciser ici ce que Camus, dans la première partie du Mythe de Sisyphe, développe: l'argument de départ du Déluge 
y fait donc référence, et pose, d'emblée l'absurdité comme acquis. Cet absurde, nous le lisons également à la fin du Procès-verbal: la question essentielle du sens dans une réalité littéralement insensée est lancée par Adam Pollo à l'équipe médicale venue l'interroger: "Vous ne voyez pas que celui qui a écrit 'la Terre est bleu comme une orange' est un fou ou un imbécile?" (ibid.: 305) C'est donc ainsi que nous envisagerons, d'abord, Le Déluge: comme une tentative de réponse à Camus, comme une tentative de retrouver, dans ce ciel vidé de sa substance, un sens, voire une foi.

\section{La faute}

Les nouvelles de La Fièvre, et plus particulièrement “L'Homme qui marche”, puis Le Déluge vont tenter de répondre à cet insensé en essayant de retrouver, dans le ciel, une possible origine, un possible "principe" si on se considère philosophe, un possible "dieu" si on se considère théologien et, surtout, une possible relation de l'homme à ce "principe". Mais à partir de quel fondement? À partir de quelle définition de l'homme? Pour Le Clézio, l'homme n'est fondamentalement ni "absurde" ni "libre”. Pour Le Clézio, encore, la rationalité cartésienne, n'est plus de mise. "Je pense donc je suis" ne peut constituer une preuve d'existence. Alors, quelle preuve? C'est peut-être dans un passage célèbre du Procès-verbal, puisque reproduit en quatrième de couverture, passage que l'on a pris souvent comme une provocation, que s'écrit un début de réponse. Dans la lettre qu'écrit Adam Pollo il mentionne "la faute de vivre". La faute n'est jamais nommée. Dans l'Avant-propos à La Fièvre, Le Clézio, provocateur aussi encore, écrit: “J’aurais préféré ne jamais naître”. Mais, la suite de la phrase où il est question de "la faute de vivre" dans Le Procès-verbal, généralement oubliée, est éloquente: “[... ] si on m'humilie, si on me fouette, si on me crache au visage, j'aurai enfin une destinée, je croirai enfin en Dieu”. Plus loin, dans ce cahier, Adam envisage la possibilité de réciter ce psaume:

Je pourrais au besoin psalmodier:

"Ce jour, jour de colère,

De calamité et de misère,

Ce jour grandiose et amer.

Quand tu viendras juger

La Terre par le feu..." (ibid.: 134)

Plus loin, encore, nous apprenons qu'Adam, avant de laisser son cahier dans les W-C du "Torpédo Snack-bar", “a signé son nom, en entier: 'Adam Pollo, martyr"” (ibid.: 228). Plus loin, encore et enfin, dans le chapitre P., Adam s'instaure en prophète et s'adresse à la foule en tant que tel. En témoigne cette formule: "Mes chers frères..." (ibid.: 243). Ces références constantes à la chrétienté ne sont pas fortuites. Elles soulignent une possible identification d'Adam aux premiers chrétiens, sous le double signe de la culpabilité et de la révélation, ce qui condamne Adam à l'incompréhension comme en atteste la retranscription de son arrestation dans le journal. 
Il y a, dans ces récurrences, une obstination quasi-augustinienne à définir l'homme par le péché, à définir l'homme comme coupable. Le patronyme "Adam" ne peut être un hasard. Nous le savons. Jusqu'où, alors, peut-on proposer une lecture augustinienne de l'homme chez Le Clézio? Saint-Augustin note que si l'homme, Adam, a été chassé du Paradis, c'est avant tout par orgueil (la superbia), pour avoir confondu le Créateur et sa Création. Notons que, jusqu'à Saint-Augustin, il n'y a aucune relation entre le péché originel et le péché de chair et que Saint-Augustin explique l'origine de l'homme à la suite de la lecture de L'Epitre aux Romains de Paul de Tharse. Le récit est l'un des plus connus au monde. Yavhé Dieu interdit à Adam et Eve de manger le fruit de l'arbre de la connaissance du Bien et du Mal sous peine, nous dit la Bible, "de mort". Relu et expliqué par Paul de Tharse, cet épisode montre que la condamnation pour la faute n'est pas ce que la glose a, plus tard, élaboré, mais ceci: la révélation à l'homme de sa propre mort.

Nous savons que par un seul homme, le péché est entré dans le monde, et que par le péché est venue la mort ; et ainsi, la mort est passée en tous les hommes, étant donné que tous ont péché. (Epitre de Saint-Paul aux Romains, chap. 5, v. 12)

Que retient, plus tard, Saint-Augustin de ces lectures? Que le péché originel est lié à cette envie du "discernement", évoqué dans la Bible, soit à l'appel de la conscience. Ce que révèle l'épisode du fruit défendu, c'est avant tout, de la part d'Adam \& Eve, la révélation à soi-même, matérialisée par la honte d'être nu, la révélation d'être à l'égal de l'animal: c'est donc la naissance de la conscience de soi. Le corollaire, la punition, est donc, dans le même temps, la révélation à l'homme de sa finitude. Hannah Arendt précise que ce mouvement de dévoilement, chez Saint-Augustin, est une perversitas qui détourne l'homme du "sens originel de son être créé, qui était justement de le renvoyer par-delà le monde à sa véritable origine." (Arendt 1995: 108). En d'autres termes, en s'attachant à la matérialité, le fruit, c'est-à-dire à la Création, l'homme se détourne du Créateur, de l'origine, et se sépare inéluctablement de la vérité. On sait également que cette pensée augustinienne s'est développée grâce à la lecture de Platon, et, plus précisément de Plotin, dans le Milan du IV siècle, et que cette idée d'une réalité scindée en un monde de l'image et d'un monde de la vérité, n'est jamais qu'une évolution de ce que le philosophe grec développe dans son mythe de la Caverne.

\section{La Conscience}

Tout lecteur assidu des premiers romans de Le Clézio a reconnu dans ce - trop bref - exposé quelques idées qui "habitent" Le Procès-verbal ou le Déluge.

L'orgueil y tient une place prépondérante. Dans cette même lettre d'Adam où il est question de la "faute", il note, avant: "je leur laisse ces ordures en guise de testament; sans orgueil, j'espère qu'on me condamnera à quelque chose...” (Le Clézio 1963: 122). L’orgueil est, si l'on croit Saint Augustin, le premier des péchés. Il est le synonyme du "discernement": de lui, découle la faute originelle, et donc, la séparation des mondes, celui du Divin, et celui qui sera désormais celui des hommes, celui du factice - c'est l'un des chapitres de 
L'Extase matérielle - hanté par la présence continue de la mort, par la conscience de la mort, enfin, comme un alpha et un oméga.

Le Clézio sait que la conscience est ce qui le définit, et est également ce qui le limite, tragiquement: "Les possibilités de l'homme sont infinies. Mais son impossibilité, sa grande et fatale impossibilité, elle, est unique" (Le Clézio 1967: 219).

Pour en arriver à cette déclaration, Le Clézio envisage d'abord la conscience comme un acte de connaissance de l'autre. Élargissant le discours freudien sur la différenciation, il pose la conscience comme principe de reconnaissance de soi à travers l'autre. Mais, note$\mathrm{t}$-il, dans cet effet de miroir, la conscience de l'autre finit par enfermer la conscience de soi, si bien qu'il ne reste de ce mouvement vers la connaissance qu'une faute, qu'une erreur: "On est spectacle pour soi comme on est spectacle pour les autres." (ibid.: 93). Ne reste alors, toujours selon Le Clézio, comme vérité se démarquant du "spectacle", que la conscience réfléchissant la conscience, comme seul instant de perfection. Pourtant, cet instant est fugace (existe-t-il?), il est destiné au Néant (ibid.: 218). L' homme est pris dans un piège: ce qui le détermine est également ce qui l'éloigne de la connaissance.

Les plus grands péchés de l'homme, il ne les commet pas à cause de ses sentiments, mais à cause de son intelligence. Il faut savoir aussi refuser le plaisir d'avoir compris, et renoncer à l'orgueil du didactisme. La foi ne vient pas parce qu'on a besoin d'elle pour être heureux (ibid.: 89).

À partir de cet instant, dans L'Extase matérielle, se lit un autre discours, augustinien, qui se développe sur l'invisible, sur cette possibilité de l'Un, platonien, puis plotinien et augustinien. C'est une interrogation.

En dehors de Dieu, impossible de faire la synthèse du tout et du rien. Lui seul est à la fois l'Objet et le Néant, lui seul possède ses propres limites. Lui seul recèle le mystère de ce qui est plus qu'être, de ce qui est arraché au terrible relief de la réalité et qui est placé dans la liberté d'être selon sa propre nature (ibid.: 219).

Accéder à ce qui est nommé ici comme "Dieu", le Dieu de Saint-Augustin, de Descartes ou de Kant, ce Dieu comme principe élémentaire ou, plutôt, y accéder à nouveau L'Extase matérielle se double d'un discours sur la connaissance de la résolution des contraires par l'homme dans un monde antécédent - afin de conjurer la peine à laquelle l'homme est condamné, la mort à laquelle Le Clézio s'adresse dans le chapitre nommé Le Miroir, devient une des grandes questions lecléziennes: il s'agit là, sans doute de la question augustinienne de la grâce. Et si L'Extase matérielle propose une solution camusienne, "à hauteur d'homme", une solution dans une forme d'acceptation, "la morale de l'impossible qu'il faut maintenant accepter" écrit Le Clézio, "pour être ajusté”, il nous semble que Le Déluge, au contraire, engage l'homme dans une autre démarche, à l'inverse de l'acceptation, une démarche éminemment - osons le mot - chrétienne.

Revenons, un bref instant, sur "L'Homme qui marche", une des nouvelles de La Fièvre, qui nous semble être l'expression la plus aboutie de l'acceptation de "la morale de l'impossible". Le personnage de cette nouvelle, J-F. Paoli se réveille, vers onze heures, après avoir avalé "une dose trop forte d'hypnogènes", et entend le bruit de l'écoulement de gouttes sur une bassine, bruit qui envahit J-F. Paoli et qui devient une terrible obsession. Ce bruit, c'est 
avant tout la conscience d'un temps régulier, imperturbable qui s'écoule "en dehors" de J-F. Paoli, d'un temps auquel il n'est pas “ajusté”. J-F. Paoli part dans la ville, part du centre pour se diriger vers la banlieue, la marge, et, au bout de quelques heures de marche qui figurent "l'ajustement" par le rythme, J-F. Paoli finit par reconnaitre le rythme de l'écoulement des gouttes entendu quelques heures auparavant dans son appartement: "C'est le rythme! c'est le rythme! j'ai retrouvé le rythme!" (Le Clézio 1965: 120).

Ce rythme, c'est celui de la "vérité" nous indique Le Clézio, c'est également, et même si la notion est difficile à définir, celui de "l'extase matérielle", principe de réunification véritable de l'homme à la matière et au Temps. Ce qui interpelle, ici, c'est ce terme de "vérité" qui devient la finalité de la démarche et de la marche. "Tout est rythme. Comprendre la beauté, c'est parvenir à faire coïncider son rythme propre avec celui de la nature." (Le Clézio 1967: 131) Cette idée d'une "vérité" sous la forme d'un "ajustement", ou d'un accord à la réalité du monde qui suppose une disparition de l'Être dans un ensemble plus grand, "cosmique", n'est pas sans rappeler les injonctions de Saint-Paul aux Ephésiens.

Au contraire, en vivant dans la vérité de l'amour, nous grandirons pour nous élever en tout jusqu’à celui qui est la Tête, le Christ.

Et par lui, dans l'harmonie et la cohésion, tout le corps poursuit sa croissance, grâce aux articulations qui le maintiennent, selon l'énergie qui est à la mesure de chaque membre. Ainsi le corps se construit dans l'amour. (Lettre 4, versets 7-16)

Sans extrapoler, nous pouvons lire dans "le rythme de la vérité" leclézien, une variation de "l'amour de la vérité" paulinien. Il s'agit d'avoir conscience de n'être qu'une parcelle d'un Tout, et d'être à sa place dans un Tout que Saint-Paul nomme le Christ. Pour Le Clézio, la réalité se substitue au Christ: pourtant, il demeure une objection notable, objection qui est au cœur du Déluge, et qui fait que Le Clézio, comme d'autres, en revient à la possibilité d'un Dieu comme "principe originel”: celui de l'origine de la réalité.

\section{Le Déluge}

À bien des égards, Le Déluge peut se lire comme une réécriture, plus ample, de "L'Homme qui marche". Le principe narratif est le même: François Besson est seul dans sa chambre. À la suite d'un "dérèglement", il est expulsé de la chambre, condamné à marcher, en ville, à la recherche d'un "ajustement" qui n'interviendra qu'à la fin du roman. Seulement, à la différence de "L'Homme qui marche", Le Déluge se double d'une recherche de l'origine, d'un sens à la réalité que Le Clézio explique à P. Lhoste en 1970. "Le Déluge c'est peut-être un projet plus ambitieux un projet de recréation de l'histoire du monde on échoue toujours quand on veut faire ce genre de chose" (Le Clézio 1971: 61).

Il faut sans doute s'arrêter en préambule sur le choix de Le Clézio de se référer au mythe du Déluge. Mythe œcuménique par excellence, le Déluge relate une punition divine (un trop grand bruit produit par les humains, dans la version la plus ancienne du mythe, celle, sumérienne, de - 1700 av J.C, celle de l'épopée de Athrahasis) qui réduit le peuplement humain à son plus strict minimum grâce à une submersion du monde. Chaque 
version mentionne un élu, un homme, prévenu par les Dieux, à qui incombe la charge de sauver la Création, Athrahasis. Dans la Bible, Athrahasis devient Noé. Dieu, cette fois, décide d'éradiquer sa création, devenue perverse et malsaine, et, après avoir averti Noé, seul Être digne, selon lui, de sa confiance, submerge l'ensemble de la Terre afin de la "laver", puis met fin à la condamnation en retirant les eaux. Dieu, alors, se repent, et fait de l'arc-en-ciel la preuve matérielle du nouvel accord qu'il noue avec les hommes.

Ce thème du châtiment par les eaux n'est pas propre aux religions hébraïques. D'autres religions ont intégré ce châtiment à leur récit: l'Avesta, le Mahabaratah, le Popol Vuh ou encore dans les mythologies scandinaves ou amérindiennes. Pourtant, le Déluge en tant que tel n'occupe pas une place importante dans le roman de Le Clézio. Certes, au chapitre VII, il est fait mention d'une tempête, certes le motif de l'eau revient plusieurs fois dans le roman, à travers le lit d'une rivière, ou à travers la sphère de l'eau sans eau, mais il n'y a pas, à proprement parler, de Déluge qui intervient. Seulement, au Chapitre IV, lors de la rencontre avec l'aveugle, il est fait mention d'un Déluge imminent qui ne viendra jamais. Il faut donc attendre la fin de la retranscription du monologue d'Anna, après la disparition de Besson, pour que le terme de Déluge soit énoncé et que le sens du titre soit dévoilé: avant de mourir après avoir avalé les "pastilles roses", celle-ci évoque une sensation proche du Déluge, et évoque Noé contemplant les eaux mortes. Ce n'est donc pas l'objet de la punition que Le Clézio a retenu. Mais quoi? Ce qu'il retient, c'est l'idée d'une perversion, d'une facticité, et d'une damnation. Ce qu'il retient aussi, c'est l'idée qu'un homme puisse être sauvé.

Arrêtons-nous désormais sur ces pages qui précèdent le chapitre 1. Elles permettent de mieux appréhender le discours leclézien sur la Faute. Elles s'ouvrent sur ces lignes: "Au commencement, il y eut des nuages, et des nuages, lourds et noirs, chassés par quelques vents, retenus à l'horizon par une ceinture de montagnes" (Le Clézio 1966: 9). L’incipit, pastichant la Bible, écrit à nouveau l'histoire d'une Genèse qui se déploie grâce à la lumière sur la réalité. Il faut ici, sans doute y voir l'influence de Plotin, le philosophe greco-égyptien qui a inspiré Saint-Augustin. La principale révolution qu'opère Plotin par rapport à Platon est que l'explication du monde n'est plus tacto-optique, comme nous l'explique G. Deleuze, mais uniquement optique. En d'autres termes, l'espace n'est pas pré-existant à la lumière soit au Temps - , l'espace devient le produit de la lumière, c'est-à-dire du Temps:

Mais il pressentait l'espèce de retournement du platonisme qu'il est train de faire. C'est avec Plotin que commence, en philosophie, un monde optique pur. Les idéalités ne seront plus que optiques. Elles seront lumineuses, sans aucune référence tactile. Dès lors la limite optique est d'une toute autre nature. La lumière fouille les ombres. Est-ce que l'ombre fait partie de la lumière? Oui, elle fait partie de la lumière et vous aurez une gradation lumière-ombre ou ombre lumière qui développera l'espace. Ils sont en train de trouver que, plus profond que l'espace, il y a la spatialisation. Ça Platon ne le savait pas. (Deleuze 1982)

La réalité est un mouvement généré par la lumière. C'est exactement ce que les premières pages du Déluge semblent corroborer: "Puis, s'amoncelaient selon les différences essentielles, une part de lumières, une part d'ombres. Les formes se réduisaient à leur expression la plus schématique, droite, spirale, angle" (Le Clézio 1966: 12). Mais, nous signale Le Clézio dès les premières lignes, ce mouvement "se faussa petit à petit". Pourquoi? La lumière, peu à peu, se diffracte, produit d'autres sources de lumière, détachées de la source première, d'autres lumières qui fracturent la réalité plutôt qu'elles ne l'ordonnent. 
Aussi, dès la page 11, Le Clézio prévient que "C'était sans importance, puisque, dès le commencement du jeu, le monde avait cessé d'être et d'avoir été." (ibid.: 11). Il en résulte la révélation d'une réalité faussée, anarchique, un "spectacle" pour reprendre le terme de L'Extase matérielle présent aussi dans Le Déluge: "C'était le monde offert en spectacle" (ibid.: 18). C'est à la page 29 que l'on trouve la description la plus aboutie de ce mouvement qui aboutit à cette conclusion: "il me semble que le monde s'est tordu ; qu'il porte une plaie incurable” (Le Clézio 1966: 31). Cette vision d'une réalité viciée, d'une réalité autant insaisissable qu'irreprésentable devient cette réalité leclézienne dans laquelle est introduit Besson, dont la singularité est celle de la conscience de la mort: le 25 Janvier, à $15 \mathrm{~h} 30$, alors que Besson regarde une jeune fille sur un cyclomoteur disparaître avec la fin d'une sirène, il déclare: "Depuis ce jour, tout a pourri. Je, François Besson, voit la mort partout" (ibid.: 21) Peut-on y lire, à la suite de ce que nous avons développé, la preuve d'une condamnation? Sans doute. Besson est l'orgueil. La conscience a révélé l'oubli, le Néant. Plus loin, p. 23, Besson écrit “C'était la damnation accordée à chaque être, pesant sur chaque chose” (ibid.: 23). Ensuite se déploie, sur plusieurs pages, l'écriture d'une réalité hantée par le mal, "Besson, figé, l'air d'une statue exotique, regarde droit devant lui et ne voit que ce mal” (ibid.: 31).

Pourtant, le dernier paragraphe de ces pages qui précèdent le chapitre 1, laissent apercevoir la possibilité de retour vers une lumière unie. "La tâche de lumière blanche" (ibid.: 32). Tel est, en définitive, l'objet de la quête de Besson: renouer avec l'unicité de la lumière avant sa fragmentation. Comme dans "L'Homme qui marche", nous l'avons dit, le départ de Besson de la chambre, où il écoute la première partie du monologue d'Anna, procède d'un dérèglement. Il ne s'agit pas, ici, ou il ne s'agit plus de gouttes d'eau et de rythme, mais de lumière. Dans cette chambre, tout est en ordre. Les objets sont identifiés, à leur place, dans l'ombre. “Tout ça était très bien. François Besson était à l'intérieur de son petit dessin calme, cerné par un cadre. [...] Une vraie caricature, où chaque chose, chaque meuble, chaque cendrier avait trouvé son contour exact" (ibid.: 66). L'immobilité, ou l'exactitude, sera troublée par un simple geste: allumer l'ampoule au plafond. Dès lors, ce qui peut être lu comme une allusion au Déluge entre dans la chambre et pénètre Besson, "une drôle de vieillesse météorologique”.

L'itinéraire de Besson le conduit à multiplier les expériences du quotidien et les expériences extraordinaires, avec sa mère, dans la rue, avec Josette, avec la femme rousse, encore, ou l'homme au mégot. Les sept premiers chapitres du Déluge relatent la déambulation de Besson dans la ville: contrairement à "L’Homme qui marche", cette progression n'est pas linéaire, du centre vers la marge, mais est anarchique, labyrinthique, fermée. Ce jumeau avoué de Le Clézio auquel il prête même un de ses premiers romans d'enfant, Oradi Noir, erre, littéralement dans une réalité devenue un piège, où toute possibilité de stabilité, d'exactitude, est anéantie au moment où elle apparaît. Ces chapitres sont aussi l'occasion, pour Le Clézio, de poursuivre cette écriture de "sismographe" du réel, inaugurée dans Le Procès-verbal et les nouvelles de La Fièvre. L'écriture ausculte cette réalité qui se déploie à mesure que Besson la regarde, cette réalité de mouvements où l'homme semble être détaché de sa conscience, enfermé dans ce "spectacle". Le "sismographe", métaphore de l'écriture, peut être envisagé également comme une volonté réitérée de l'écrivant de comprendre la réalité afin, peut-être, de l'appréhender et de la dompter de façon quasi-tellurique ce qui sera effectivement le cas, plus tard en 1986, sur l'île Rodrigues lorsqu'il s'agira de capter 
une réalité du passé, celle son grand-père. Revenons au Déluge. Le chapitre VIII marque une rupture. C'est celui de la tempête, celui du Déluge.

Au début du chapitre, Besson reste dans sa chambre. Ironiquement, il écoute, à la radio, une annonce publicitaire qui se sert du Déluge pour vanter les bienfaits d'une crème hydratante: tel est, dans ce monde du "factice", l'appréhension de l'homme moderne du châtiment. Plus tard, Besson décide de sortir dans la ville, seul, au milieu du déferlement. La réalité urbaine prend alors une autre forme: "Mais il ne s'occupait plus de rien; les vitrines des magasins, et les miroirs étaient devenus, non pas plus opaques, mais plus clairs tellement plus clairs qu'on aurait dit qu'en eux étaient la réalité" (ibid.: 166). L'utilisation du conditionnel renforce l'écriture d'un dévoilement, pour Besson, à ce moment-là, celui d'une Lumière originelle qui redonne à ces objets des hommes une forme de vérité. Besson, ensuite, s'avance vers la plage, vers ce qui semble être l'origine du Déluge. Après quelques pages de description - qui mériteraient un commentaire particulier - du Déluge et de la progression de Besson dans ce Déluge, ce dernier trouve refuge en son centre, matérialisé par le phare. Notons - est-ce un hasard? - que Le Clézio emprunte le même décor que Beckett dans La Dernière bande pour la "révélation": la nuit, la digue, le phare, la tempête... Dès lors, Besson fait une expérience cosmique, la même que J-F. Paoli, mais dans un environnement plus "ambitieux": le Déluge devient un corps possédant une respiration qui lui est propre, et Besson fait connaissance, non pas avec sa Douleur, mais avec l'Eternité: "Et le cycle de la respiration recommençait comme avant, sans fatigue, sans hâte. Besson sentit qu'il était en train d'entrer en quelque sorte dans l'éternité" (ibid.: 173). Une page plus loin, se lit alors l'acmé de cette séquence, soulignée par l'utilisation de l'italique: "C'était cela le secret de la vie éternelle. Respirer. Ne jamais s'arrêter. Respirer avec le reste du monde. Respirer dans la mer, respirer au cœur des rochers, dans les limbes des nuages, au milieu du vide noir où avancent les galaxies. Respirer selon le rythme de la vérité" (ibid.: 173-174). Nous retrouvons ici cet avatar de la devise paulienne qui conçoit Une vérité au cœur du réel, matérialisée par un rythme, par un Temps, universels. Retrouver le chemin de l'Eternité, retrouver, donc, le chemin du réel revient à se démarquer des Temps fragmentés, pour se retrouver dans ce Temps de l'Un, à se confondre dans l'Un, dans la Création première. Il est donc normal, au terme de la séquence du Déluge, qu'intervienne un signe du Divin, "le signe divin et fulgurant de la blancheur" (ibid.: 175) par le biais du tonnerre.

Si Le Déluge en était resté là, il n'aurait été que réécriture de "L'Homme qui marche". Ce sont dans les trois chapitres suivants que Le Clézio donne une évolution nouvelle à son récit, et qu'il s'attache, désormais, au problème augustinien de la grâce. Le chapitre IX voit Besson, après cette révélation s'enfuir, sans raison. Marthe lui demande "Pour quoi faire?" "Pour rien, dit Besson" ce à quoi Marthe lui répond: "Pourquoi, pour rien?”. En effet, sous l'apparent humour du dialogue, se dissimule, peut-être la raison profonde de cette fuite: se sauver du Rien, du Néant dans lequel le monde est retombé. Car tel est, désormais, la position de Besson. Il est le seul homme qui sait. Il est, désormais, Noé, en charge de sauver l'humanité. Dit ainsi, nous pourrions croire qu'il s'agit d'une extrapolation. Pourtant, il n'en est rien. La question est, après cette révélation par le Déluge où, seul Besson fut "touché" par la lumière, comment vivre dans un monde où, pour les autres, rien n'a changé, où tout est resté dans le "spectacle"? La lecture des sous-titres des chapitres IX, X, XI \& XII est édifiante: Besson erre seul, désormais. "François Besson connaît la faim, la soif et la solitude/ François Besson offre sa nuque à Dieu/ La confession/ Comment Besson apprit 
le métier de mendiant/ François Besson au bagne etc." Besson devient ce qu'Adam Pollo avait annoncé être: un futur Martyr. Dans ce parcours post-Déluge, nous reconnaissons un itinéraire quasi-christique, où, après les quarante jours dans le désert, après l'ascèse, après l'expérience de la dépossession de soi (Besson se livre d'ailleurs à un auto-dafé de tous ses écrits, au chapitre IX, avant de quitter définitivement la maison de ses parents), reste le sacrifice de l'homme pour les autres.

Parmi ces séquences, une est éminemment significative. Au chapitre X, Besson se rend dans une église et, après avoir "offert sa nuque à Dieu", il se dirige vers un confessionnal. Sur quatre pages, Besson récite l'ensemble des péchés qu'il a commis. Aucun ne manque. Et lorsque le prêtre insiste Besson répond ceci: “J’ai voulu trop savoir. J’ai cru - J’ai oublié la Vérité. J’ai oublié.” (ibid.: 205). Lorsque le prêtre demande à Besson quelle faute il regrette, il répond ceci: "Le - L'orgueil. Et le mensonge, le blasphème." (ibid.: 206). Le commentaire est dispensable. C'est donc en des termes augustiniens que Besson reconnaît son péché. La confession finie, le prêtre se lance dans une leçon de théologie, là encore, augustinienne. "Il faut vous humilier, mon fils", dit-elle. "Humiliez-vous dans votre corps et dans votre esprit. Renoncez à ce qui est futile. Tuez votre orgueil." (ibid.: 207). Et à la question de savoir si Dieu existe, le prêtre de répondre qu'il n'est pas absent, mais Besson ne sait pas le voir. Ce qui étonne, dans ce discours du prêtre, c'est qu'il reprend, au mot près, le développement d'Adam à la fin du Procès-verbal ou certaines pages de L'Extase matérielle, résumées dans cette interrogation: "Ne savez-vous pas que l'intelligence ne vous sert à rien?” (ibid.: 207). Sacrifier l'intelligence, la conscience réfléchissant la conscience, le cogito leclézien devient, en définitive la seule « porte de salut » ce qui était annoncé par Adam, juste avant son retour sur soi: "De n'importe quelle autre façon, la connaissance aboutit à une impasse.” (Le Clézio 1963: 298).

Besson suit les indications du prêtre. La mendicité est vue comme une tentative de "pénitence", puis le repas au Salut Public, puis la dernière cigarette. Ensuite, au chapitre XI, Besson entreprend de travailler comme terrassier: un travail de force qui n'est pas sans rappeler le "tripaliare" latin qui renvoie à la torture. Enfin, à la fin de ce même chapitre, il y a cette autre séquence, celle du meurtre de l'inconnu où Le Clézio y réinterprète, librement, la tentation opérée par le Diable sur Jésus lors de ces quarante jours dans le désert. Quel est cet inconnu que Besson tue à coups de caillou? Là encore, les deux pages de l'attaque mériteraient une analyse approfondie. Cet inconnu est tout d'abord un diable, perçu sur le bord de la rivière, en pleine nuit. Tout d'abord "loup" ou "fauve", résurgences d'une représentation moyenâgeuse de la Bête, Besson l'identifie ensuite comme un homme, "très noir", doté d'une odeur forte, l'homme devient rapidement un "monstre" qui, symboliquement, prend les dimensions de la ville.

Véritable figure du Mal, l'homme incarne ce que l'espace urbain est devenu pour Besson: un Mal, là encore augustinien, une "habitude" qui attache l'homme dénué de volonté à la Création et non au Créateur pour oublier la Mort. Comme le souligne Hannah Arendt, le Mal, chez Saint-Augustin n'a rien de fantastique, il est l'absence du Bien, l'oubli du Bien. La ville, telle que la conçoit Le Clézio, par les yeux de Besson, est marquée par cet oubli, par cette habitude dans une réalité coupée à jamais de son origine. Besson est, désormais, le seul à le savoir. Le meurtre accompli, Besson s'enfuit par les égouts pour accéder à la lumière de l'autre côté de la ville. Nous y lisons alors cette phrase: "À l'intérieur du cylindre fermé, où jamais la lumière du jour ne venait, il se lança en avant, tout son corps offert et 
douloureux, comme une parcelle de la raison et de la sagesse au milieu del'âcre océan de folie." (Le Clézio 1966: 221).

Le chapitre XII voit Besson s'enfuir, monter dans un car et y descendre en pleine campagne et poursuivre son chemin à pied jusqu'à une étendue déserte où il s'installe "sur un gros caillou au bord du chemin". Dès lors deux séquences s'enchaînent, l'une la contemplation d'un oiseau dans le Ciel, l'autre, la décision de retourner ses yeux vers le soleil, vers la lumière. Cet oiseau qui vole est le premier d'une longue série, chez Le Clézio, dont le plus célèbre est l'aigle, dans Désert. Véritable symbole du vivant en relation avec le Créateur, lors de sa description, la phrase devient nominale, simple, et l'oiseau devient le symbole d'une Éternité retrouvée, "abandonné à l'infinité du dessin horizontal" (ibid.: 243). Ce passage permet à Besson d'apercevoir, enfin, ce qu'il recherchait: "Le rideau de pluie et des nuages s'était déchiré soudain, ce jour-là, dévoilant la nudité du ciel et la rondelle fulgurante du soleil." (ibid.: 247). Besson, en tant qu' homme, lutte un instant: l'habitude, le souvenir revient en lui, en pure perte, Besson est prêt à rejoindre la lumière:

Et l'éternel est là ; non pas caché, mais omniprésent. Non pas recouvrant tout, mais à l'intérieur, au centre du centre du temps.

Alors, quand Besson connut cette grande beauté. (ibid.: 249)

La quatrième de couverture envisage Besson comme un nouvel Ædipe. Il n'en est rien. Ce moment de joie pure, où la lumière descend en Besson pour nettoyer "tout jusqu'au fond de son cerveau" (ibid.: 251), est un moment de révélation, de réconciliation. Besson se relie au Créateur, à ce soleil qui intriguait tant Plotin et Camus. S'il est OEdipe, c'est peut-être par cet acte de soumission, qui est un acte de grâce pure, par lequel il n'accepte pas son Destin, mais par lequel il accepte sa dissolution dans un Corps plus grand, dans un Ordre plus grand.

Mais, et c'est le sens, du chapitre final "Le Silence”, cela n'est qu' illusion. Le Clézio sait que ce double, ce Besson martyre, ce Besson qui donne sa vie pour rejoindre l’Un, commet un acte qu'aucun homme, ni lui-même, ne peuvent se permettre. Dans ce chapitre, Besson redevient ce qu'il est, un cadavre qui a vécu dans l'habitude, dans le "factice, dans la T.V" pour reprendre les termes du début du prêche d'Adam dans Le Procès-verbal. Alors que retenir de cet impossible qui s'est écrit?

\section{Conclusion}

L'objet de cet article n'est pas de démontrer que Le Clézio est un écrivain “chrétien” au sens où purent l'être Claudel ou Bernanos. Mais, et contrairement à ce que soutient Le Clézio, dans l'entretien avec Justyna Gambert, ses premiers romans témoignent d'une plus grande proximité qu'on ne le pense avec la mystique chrétienne, celles des premiers chrétiens et non pas celle, plus tardive de Thomas D’Aquin. Le Clézio l'admet à demi-mots lorsqu'il déclare: "Peut-être qu'elle [la mystique chrétienne] n'est pas fondamentalement différente [de la mystique paganiste]" (Le Clézio 2015: 68). En effet, l'idée de l'Un, d'un principe Créateur, qu'elle soit pure lumière, comme chez Plotin, ou d'origine divine, chez SaintAugustin, l'idée que chaque être puisse être une parcelle de cet Un et le discours qui en 
découle, toujours chez Saint-Augustin, sur le péché et la rédemption ne sont pas si éloignés, en définitive des autres religions que Le Clézio, plus tard, abordera. Ces idées, si elles lui sont familières, c'est avant tout parce qu'elles sont le produit du monde Grec, le produit d'une Méditerranée où Platon conduit à Plotin, Plotin à Saint-Augustin, Plotin à Camus, et à Le Clézio.

Besson, nous l'avons vu, est le double de Le Clézio, écrivain, non pas par profession, mais par nécessité: il octroie à Besson un récit d'enfance, Oradi Noir, Besson travaille à divers écrits, au début du Chapitre V., et brûle ces mêmes écrits. Plus tard, Le Clézio confiera à $\mathrm{M}$. Butor vouloir le faire lors d'une rencontre à Nice. Le Déluge parle donc, aussi, du rôle de l'écrivain tel que le conçoit Le Clézio, à rebours des idées de la modernité. Dès cette époque, et cela est sans doute la source de beaucoup de malentendus, Le Clézio réclame pour l'écrivain un rôle archaïque, premier, proche des premiers théologiens, interrogeant le mystère du ciel. De ceci, il nous en avertit dès L'Extase matérielle:

Toute littérature n'est que pastiche d'une autre littérature. En remontant ainsi dans le temps, jusqu'où arrive-t-on? [...] La continuité est un tout. [...] La véritable notion humaine, celle qui finalement se rapproche le plus de ce qu'on conçoit aussitôt de la vie, c'est l'éternité. (Le Clézio 1967: 126)

Cette théologie, c'est celle de Saint-Augustin où la pensée est avant tout une volonté de vérité. Certes, Le Clézio n'ira sans doute pas à considérer que la vérité est Dieu, mais il y a chez le Nobel 2008, une constance à penser qu'il existe une vérité, l’Un, dont il a cherché les preuves chez les Embreras, dans les codex amérindiens, dans les pierres de Rodrigues ou sous le ciel de Séoul, une constance à écrire une vérité, l'Un, qu'il nomme Es Ser, Argo ou Aro Chuku, un dessin du ciel, parfait, une "mécanique céleste", pour reprendre un terme cher aux astronomes normands, Laplace et Le Verrier, à laquelle l'homme doit s'accorder afin de répondre à cette injonction de Saint-Paul contre "l'absurde":

Je vous le dis, j'en témoigne dans le Seigneur: vous ne devez plus vous conduire comme les païens qui se laissent guider par le néant de leur pensée. (Lettre 4, verset 17)

\section{Bibliographie}

Arendt, Hannah 1995. Le Concept d'amour chez Saint-Augustin. Paris: Rivages.

Deleuze, Gilles 1982. “Spinoza, cours n¹0, du 17/01/1982”. http://www2.univ-paris8.fr/deleuze/ article.php3?id_article=21 [09.11.2019].

Le Clézio, Jean-Marie Gustave 1963. Le Procès-verbal. Paris: Gallimard, coll. Le Chemin.

— 1965. La Fièvre. Paris: Gallimard, coll. Le Chemin.

— 1966. Le Déluge. Paris: Gallimard, coll. Le Chemin.

— 1967. L'Extase matérielle. Paris: Gallimard, coll. Le Chemin.

- 1971. Conversations avec Pierre Lhoste. Paris: Mercure de France.

Paul de Tharse 2008. Epitres de Saint-Paul: traduction officielle. Paris: Desclée de Brouwer.

Saint-Augustin 1998-2002. Ouvres. Paris: Gallimard, coll. La Pléiade. 